\title{
Top challenges of LTE to become the next generation railway communication system
}

\author{
J. Calle-Sanchez, M. Molina-Garcia \& J. I. Alonso \\ Department of Signal, Systems and Radiocomunications, ETSIT, \\ Technical University of Madrid, Spain
}

\begin{abstract}
Railway environments cannot be away from the current evolution of public communication systems. The Long Term Evolution (LTE) mobile communication system is considered to be the natural evolution for current Global System for Mobile Communications Railways (GSM-R). LTE all IP networking technology plays a key role for the integration and convergence of heterogeneous networks and services, both the specific for the railway operative and the commercial broadband ones for passengers. The adoption of a new generation standard for railway communication must be based on an exhaustive feasibility study of the new system to become the railway mobile communication system. In this paper, LTE's top challenges to address the specific requirements and features of railway communication services are evaluated. Under this scope, LTE features to implement the required railway functionalities, LTE technical challenges, spectrum harmonization, network deployment considerations and LTE capabilities to meet the railway service Reliability, Availability, Maintainability and Safety (RAMS) requirements, are assessed.

Keywords: LTE, high speed railway, VoLTE, LTE features, RAMS, challenge assessment.
\end{abstract}

\section{Introduction}

The need to upgrade existing railway mobile radio systems can be linked to both technological and economic factors. The future radio system, must offer substantial benefits to train operators and infrastructure managers. The technology upgrade process has a high economic impact in terms of deployment costs. 
Technological reasons to change the GSM-R railway telecommunication system are linked to the improved performance and capacity of new communication systems, and GSM-R end of life cycle (Mandoc [1]). In the midterm roadmap of railway communication systems evolution, the support and maintenance of the current GSM-R system will not only be outdated but also expensive. Moreover, the future train radio system advanced features and capabilities will allow railway operators to support a strong quality of service demanding applications and added value services. Therefore, these advanced features will play a key role in the provision of advanced passenger services, signaling applications or operational services (Furuskar et al. [2]).

Regarding the economic factors, these new communication technologies can generate opportunities to increase railway operator's revenues by means of new added value applications and services, as well as reducing operational and maintenance costs. This reduction can be achieved by means of the efficient operation of the operator's rolling stock based on real time information and improved communication between moving trains, maintenance staff and trackside systems (Barbu [3]). Furthermore, communication and signaling systems were custom-made for railway environments which derive into an increased high-cost development process. Therefore, railways operators and infrastructure managers shall look for the cost reduction achieved in public commercial communication deployments by means of a much larger mass market.

The future radio communication system must meet all the railway specific needs and requirements. Those specific needs and requirements are related to RAMS requirements, spectrum harmonization, end to end QoS requirements, system performance in high speed scenarios, communication system deployment strategy, and system backward compatibility as well as the future system features for delivering railway services.

In this paper, the challenges associated with current railway communication systems migration towards a potential future wireless system, are assessed. Since LTE is considered to be the natural evolution for current GSM-R system in high speed railway environments, LTE top challenges to address the specific requirements and needs of railway communication services must be assessed.

\section{Railway services}

Railway services may be classified into core services and additional communications services. The core services are usually referred to as mission critical services, and include critical railway communications, train operational voice services and operational data applications. The additional communication services include passenger experience services and all the business process support services such as voice and data train crew communications and train support applications (Wootton [4]). The classification of railway group services into different services is shown in fig 1 .

The railway core and additional communication services are supported by both fixed and radio networks. Since the 1990s, digital wireless train communication systems are gaining momentum to replace the legacy railway 


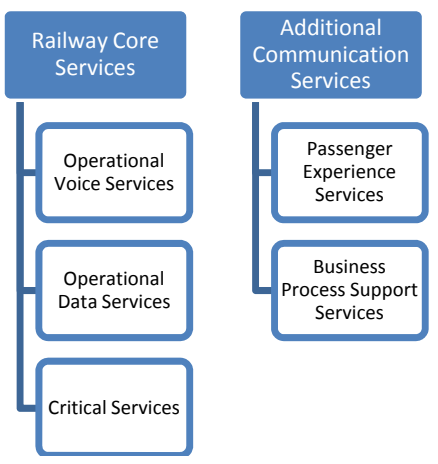

Figure 1: Classification of railway services.

networks. The railway radio systems like GSM-R, support critical mobile voice and data communications for train drivers and line-side workers. The future added value railway services demanded by operators and infrastructure managers, exceeds by far the capacity of GSM-R (Barbu [3]). A comprehensive list of the future services in train environments is shown in table 1.

Table 1: $\quad$ Expected future railway services.

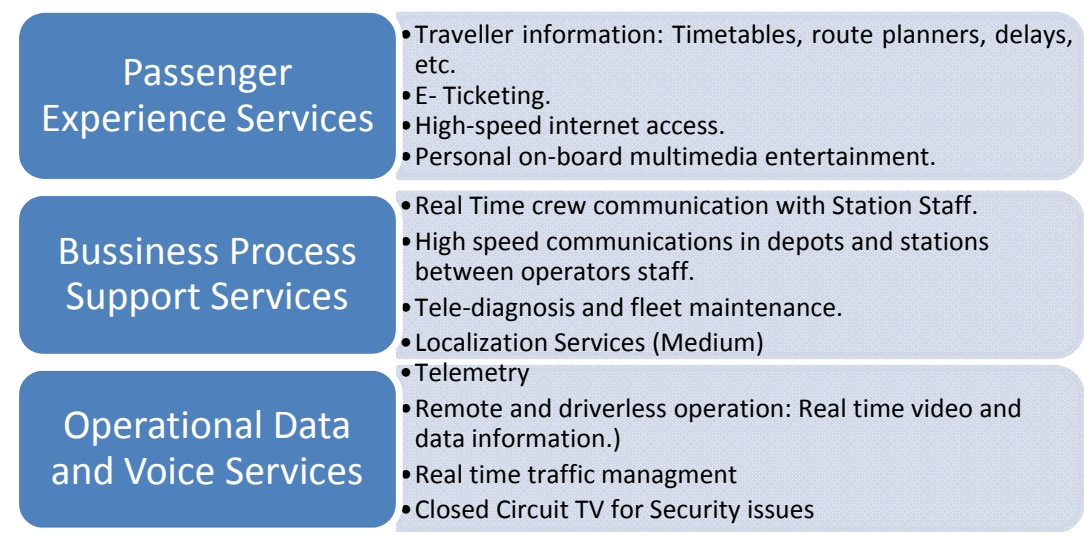

Although new requirements and functionalities could arise from new services and applications in railway environments, at least, the future train radio systems will have to fulfil the specific RAMS and QoS requirements demanded by railway services (Mandoc [1]). In addition, there is a mismatch between core services and additional services QoS requirements. Mission critical services demand stronger delay, reliability, availability and safety assurances, while the additional services requirements are mainly based on the bandwidth capacity.

This mismatch plays a key role in the deployment strategy of train digital radio infrastructure. Operators and infrastructure managers shall decide between deploying a private radio system only for railway purposes, or a hybrid solution based on a shared deployment with a public telecommunication company. 


\section{Communication systems in railway environments}

Specific requirements of core and additional communication services demand several key railway functionalities that must be supported by the train radio communication systems. Some of these functionalities may not be implemented in the communication system and must be added to the standard functionalities (Ljubic and Simunic [5]). Therefore, future railway communication system features to support the specific existing and expected future railway required functionalities must be assessed.

As described above, the train radio system must also fulfil the specific QoS and RAMS requirements demanded by the railway services. For instance the European Rail Traffic Management System (ERTMS) service demands high levels of service reliability and availability, which is translated into low call drop rates or low connection establishment failure probabilities. Therefore, these specific technical requirements must be met by the future system features when delivering core railway services.

\section{Top challenges for the future railway communication system}

In this section, top challenges for the LTE system to become the future railway communication system, will be identified and discussed. These challenges are related to the LTE mechanisms and features to implement the required railway functionalities, LTE technical challenges, the convergence to an all IP network, spectrum harmonization, network deployment considerations and LTE capabilities to meet the service RAMS requirements.

\subsection{LTE features for supporting required railway functionalities}

Railway services demands specific functionalities to train radio systems. For instance, GSM standard was enhanced with the ASCI functionalities. Proposed LTE features and mechanisms to implement the railway functionalities are shown in table 2.

The IP Multimedia Subsystem (IMS) is enhanced-services architecture for delivering any service, reaching any customer regardless of how they connect to the network (Badard et al. [7]). The main drawback is the fact that it is far from ready for deployment. The Session Initiation Protocol (SIP) is a protocol that facilitates the formation, modification and execution of communication sessions between individual or multiple participants. Locating call recipients and talking with them on their different user agents is accomplished using a SIP address, Chen [8]. The Push to Talk Over Celular (PoC) deployed into an IMS Service Delivery Platform can be an efficient way to provide the VGCS functionality in LTE standard while fulfilling railway services delay requirements. However, Voice Group Call Services (VGCS) is radio cell based in contradiction to PoC functionalities (Mandoc [1]). 
Table 2: $\quad$ Proposed LTE features to support GSM-R railway functionalities.

\begin{tabular}{|c|c|}
\hline GSM-R Functionality & LTE Feature \\
\hline $\begin{array}{l}\text { Voice Group Call Service } \\
\text { (VGCS) }\end{array}$ & $\begin{array}{c}\text { LTE IMS based VoIP (VoLTE) + IMS } \\
\text { based Push to talk Over Cellular (PoC) }+ \\
\text { Enhanced Multimedia Broadcast Multicast } \\
\text { Service (eMBMS) }\end{array}$ \\
\hline Voice Broadcast Calls (VBS) & $\begin{array}{c}\text { VoLTE }+ \text { PoC and/or eMBMS: IP multicast } \\
\text { of voice and video services }\end{array}$ \\
\hline $\begin{array}{l}\text { Priority and Pre-emption } \\
\text { (eMLPP) }\end{array}$ & $\begin{array}{l}\text { Access Class Barring mechanisms + Policy } \\
\text { Control Rules + QoS mechanisms (ARP). }\end{array}$ \\
\hline Functional Addressing (FN) & Session Initiation Protocol (SIP) Addressing \\
\hline $\begin{array}{l}\text { Location Depending Addressing } \\
\text { (LDA, eLDA) }\end{array}$ & Localization Services in LTE (Release 10) \\
\hline $\begin{array}{c}\text { Railway Emergency Calls (REC, } \\
\text { e-REC) }\end{array}$ & $\begin{array}{c}\text { Emergency and critical safety voice services } \\
\text { over IMS in LTE. }\end{array}$ \\
\hline Fast Calls Set-up & IMS based PoC + Access Class Barring \\
\hline Data Exchange (SMS, Shunting) & IMS based SMS Service \\
\hline
\end{tabular}

Voice service provision, priority and pre-emption mechanisms, and QoS and Access Barring mechanisms, will be further described in the LTE technical challenges section.

\subsection{Technical challenges of LTE}

In this section, main technical challenges of LTE for supporting railway functionalities with their specific QoS requirements are described.

\subsubsection{Voice service provision over IP LTE networks}

GSM-R functionality for delivering voice services is considered a key core functionality for railway operation. The voice service provision in the LTE standard is a major challenge that must be carefully assessed and analyzed. Regarding the technological solutions for delivering voice over LTE, the solutions based on using the IP Multimedia Subsystem (IMS), the hybrid voice over LTE via Generic Access (VoLGA) solution and the CSFB solution are the most promising ones (Paisal [9]). However, it seems that the IMS based solution known as One Voice Profile for Voice over LTE (VoLTE) is gaining momentum. The main characteristics of each solution and the advantages and disadvantages from the railway point of view, are defined in table 3 .

\subsubsection{Handover in LTE}

LTE standard support hard handover mechanisms, which reduces the complexity of the LTE network architecture. However, the hard-handover (HHO) mechanism does not guarantee any data packet losing in handover process. LTE HHO must fulfil the railway service QoS and RAMS requirements, especially in high speed scenarios. It is necessary that the HHO mechanisms supported in LTE, lossless and seamless, minimize the packet loss or avoid it completely with fast connection and re-association time. 
Table 3: $\quad$ Solutions for voice service provision over LTE.

\begin{tabular}{|c|c|c|c|}
\hline VoIP Solution & VoLTE (IMS) & VoLGA & CSFB \\
\hline $\begin{array}{c}\text { 3GPP } \\
\text { Standard } \\
\text { approved }\end{array}$ & Yes & No & Yes \\
\hline $\begin{array}{c}\text { Seamless } \\
\text { Continuity of } \\
\text { Voice Services } \\
\end{array}$ & $\begin{array}{l}\text { Single Radio-Voice } \\
\text { Call Continuity } \\
\text { Mechanisms [10] }\end{array}$ & $\begin{array}{c}\text { Single Radio-Voice Call } \\
\text { Continuity Mechanisms [10] }\end{array}$ & $\begin{array}{l}\text { Fallback to legacy } \\
\text { circuit switched } \\
\text { GSM-R network. }\end{array}$ \\
\hline $\begin{array}{l}\text { SMS Support } \\
\text { Technology }\end{array}$ & $\begin{array}{l}\text { SMS over IP } \\
\text { Functionality (IP- } \\
\text { Short-Message- } \\
\text { Gateway) } \\
\end{array}$ & $\begin{array}{l}\text { VoLGA Network Controller } \\
\text { (VANC) Interface for } \\
\text { delivering all Circuit } \\
\text { Switched Services. } \\
\end{array}$ & SMS over SGs \\
\hline $\begin{array}{l}\text { Support of } \\
\text { IMS Based } \\
\text { Services } \\
\end{array}$ & $\begin{array}{l}\text { Support only IMS } \\
\text { Based Services }\end{array}$ & $\begin{array}{c}\text { Support IMS and } \\
\text { Combinational Services } \\
\text { (CS+IMS) over LTE }\end{array}$ & $\begin{array}{l}\text { No support of any } \\
\text { IMS Service }\end{array}$ \\
\hline $\begin{array}{c}\text { Main } \\
\text { Advantages for } \\
\text { Railway } \\
\text { Operation }\end{array}$ & $\begin{array}{l}\text { Supports simultaneous } \\
\text { voice/ data over LTE } \\
\text { Low service latency } \\
\text { Reduced Call Setup } \\
\text { times } \\
\text { Support of LTE QoS } \\
\text { mechanisms. } \\
\text { Supports handover } \\
\text { between LTE and } \\
\text { GSM/UMTS and vice } \\
\text { versa. } \\
\text { Provides end to end IP } \\
\text { multimedia services. }\end{array}$ & $\begin{array}{c}\text { Supports simultaneous voice/ } \\
\text { data over LTE } \\
\text { Reduced Call Setup times. } \\
\text { Support of LTE QoS } \\
\text { mechanisms. } \\
\text { Supports handover between } \\
\text { LTE and GSM/UMTS and } \\
\text { vice versa. } \\
\text { No MSC upgrades }\end{array}$ & $\begin{array}{c}\text { No IMS } \\
\text { Infrastructure } \\
\text { Required. } \\
\text { Relative low } \\
\text { initial Capex } \\
\text { Ready to Apply } \\
\text { Supports } \\
\text { handover of active } \\
\text { calls between LTE } \\
\text { and GSM/UMTS }\end{array}$ \\
\hline $\begin{array}{c}\text { Solution } \\
\text { Drawbacks }\end{array}$ & $\begin{array}{l}\text { IMS infrastructure } \\
\text { must be deployed and } \\
\text { integrated } \\
\text { Into the operator's } \\
\text { network. } \\
\text { High Initial Capex }\end{array}$ & $\begin{array}{l}\text { VoLGA is not standardized } \\
\text { in 3GPP yet. } \\
\text { Railway services that rely on } \\
\text { an end-to-end IP path will } \\
\text { not work. } \\
\text { Limited operator support } \\
\text { Generic Access based dual } \\
\text { mode mobile phones are } \\
\text { required }\end{array}$ & $\begin{array}{l}\text { Additional call } \\
\text { set-up latency } \\
\text { (from } 1 \text { to } 8 \\
\text { seconds) } \\
\text { Fall back to } 2 \mathrm{G} \\
\text { suspend LTE data } \\
\text { transmission. } \\
\text { Fall back to } 3 \mathrm{G} \\
\text { reduce data } \\
\text { transmission. }\end{array}$ \\
\hline
\end{tabular}

There are two approaches for improving handover failure rate while minimizing handover time in high speed environments. First possibility is to implement Soft Handover (SHO) mechanisms for LTE networks, which imply the mobile terminal to be connected two eNodeB simultaneously. SHO mechanisms demands larger spectrum resources and system throughput, but it has a better performance in terms of delay and packet loss. The complexity of soft handover implementation and the frequency resource consumption must be assessed for railway environments (Qiwei et al. [11]). 
The second approach is focused on optimizing LTE HHO mechanisms. Those mechanisms must minimize packet losing, while reducing $\mathrm{HHO}$ times and fulfilling maximum delay times for packet transmission. Requirements in terms of handover time are a key challenge due to the frequent handovers in railway high speed environments (Luan et al. [12]). For this purpose, handover parameters such as handover triggers in IDLE and Connected states and/or RSRP and RSRQ thresholds must be adjusted.

\subsubsection{Quality of service mechanisms and access control in LTE networks}

Service prioritization, the ability to pre-empt users, and Quality of Service are all crucial to a future railway communication system. In railway environments, LTE must assure the delivery of data and voice packets while meeting a combination of delay, jitter, dropped call rate or data error rate, handover interruption time, maximum call setup time and maximum/guaranteed bit rate requirements. Real time scheduling mechanisms in LTE for resource assignation according to user priorities and service categories are a key element for assuring railway services QoS and RAMS requirements. The key LTE features to support the required railway functionalities are described next (Sesia et al. [13]).

\section{- $\quad$ End to end QoS mechanisms.}

LTE QoS architecture is based on a bearer system. The Traffic Flow Template (TFT) is a set of packet filters associated with an Evolved Packet System (EPS) bearer. The bearers can be default or dedicated, and have 4 configurations parameters; QCI (QoS class identifier), ARP (Allocation and Retention Priority), GBR (Guaranteed bit rate), AMBR (Aggregate Maximum Bit Rate). The suitable configuration of this set of parameters, for each one of the railway services, must be carefully evaluated to meet current GSM-R requirements.

The ARP parameter is stored in the Home Subscriber Profile (HSS) and it indicates the user priority level, as well as the pre-emption capability and vulnerability. ARP Control mechanisms can deny a radio bearer request or preempt an existing one, and accepting the new radio bearer request. This is a key parameter for implementing the pre-emption functionality.

An EPS bearer is established throughout several interfaces, therefore the bearer QoS parameters must be mapped into each LTE interface QoS parameters. For instance, the mapping of radio bearer QoS parameters into transport network MultiProtocol Label Switching (MPLS) parameters must be carefully assessed.

- $\quad$ Real time resource scheduling depending on user access category.

The MAC scheduler is in charge of radio resource dynamic assignation depending on bearer QoS levels and Channel Quality Indicators (CQI) parameters, on UE radio conditions, on the different EPS bearer's status and on the interference levels between adjacent cells. 
- Dynamic access control.

The access control mechanisms in LTE are implemented both in the radio access interface and in the Evolved Packet Core (EPC) of LTE network. The access control can be implemented by establishing access classes. There are 15 access classes defined in the LTE standard, with different ARPs values to define different user categories for railway environments.

For radio interface, the main mechanism is the Access Class Barring (ACS) (Sesia et al. [13]). For the core network, the access control is implemented by means of the policy charging and control (PCC) functionalities, which are listed next:

- Policy control. The policy control supports several features to control QoS, packet forward and for the EPS bearers establishment.

○ IMS emergency session support. This feature can support the emergency calls in railway environments, as it has been stated before.

- Service Prioritization. This feature is concerned to guarantee that mission critical services in railway environments continue their operation, if the network capacity is lowered.

- Access class barring. The access class barring mechanisms control the cell selection and reselection procedures. Cell-Barring prevents user from select or re-select cells, even in emergency calls. Cell-Reserved, reserves cells for operators activities and only access classes from 11 to 15 are allowed for cell selection or reselection. This feature allows deploying a mixed network with a public commercial operator, reserving some cells for railway purposes only.

\subsubsection{Network performance in high speed environments}

The impact of high speed in LTE performance and capacity should be assessed carefully. The most important issues that should be evaluated are:

\section{- $\quad$ The Doppler shift effect in LTE downlink and uplink channel performance.}

The subcarrier interval of OFDM in LTE standard shall allow avoiding the InterCarrier Interference (ICI) impact on LTE performance but this issue must be evaluated in high speed scenarios (Ren et al. [14]). The implementation of additional ICI strategies should also be evaluated.

\section{- $\quad$ The effect of high speed in resource scheduling.}

The radio resource management functionalities optimize the radio resource assignation by means of algorithms that schedule the channel use both in time and frequency domains. This scheduler process is based on the control signalling information provided by the user equipment in terms of ACK/NACK and channel state reports (CQI). In a high speed environment, the channel measurements for channel estimation may not be able to adapt fast enough to channel variations, and schedulers also loss the diversity gain, which decrease system performance (Nochev et al. [15]). 
- Handover mechanisms in LTE.

Handover mechanism performance in high speed environments must be assessed. Depending on evaluation results, other types of handover mechanisms like soft handover ones to avoid packet loss while minimizing handover interruption time, can be implemented. Otherwise, it may be necessary to optimize the seamless HHO mechanisms in LTE to reduce connection and reassociation time while minimizing the handover failure rate in high speed environments.

\subsection{Convergence to an all IP network}

The convergence to an all IP network will provide a more efficient use of the limited capacity at all the LTE network interfaces. This convergence process may also reduce implementation and operation costs of both components and systems.

Main drawbacks and challenges of this convergence process are the QoS assurance mechanisms in IP methods, guaranteed call setup times, proper service prioritization and IP security issues. The LTE QoS mechanisms have already been discussed and they are likely to fulfil railway requirements. Regarding the call setup times, it depends on the chosen LTE VoIP mechanism, and in the IMS signalization service mapping into QoS requirements.

\subsection{RAMS}

The design of communication systems for railway environments is conditioned by the specific requirements of railway services, especially the core ones. These requirements are analyzed considering several standards that quantify and assess the reliability, availability, maintainability and the safety requirements for railway communication systems. Therefore, it is necessary to evaluate LTE capabilities and methods to meet RAMS requirements in railway environments [16].

\subsubsection{Reliability and availability}

Reliability and availability concepts are closely related. Reliability requirements for the radio link access depend on a good radio coverage planning and dimension of the LTE network. This is a difficult task, due to the complexity of railway environment, which includes bridges, viaducts, tunnels (Briso-Rodriguez et al. [17]). To overcome this challenge, a specific radio planning software that takes into account not only the environment but also the features and capabilities of LTE standard, Adaptative Modulation and Coding (AMC), HARQ mechanisms, MIMO capabilities or short TTI values, should be employed.

Moreover, Self Organizing capabilities of LTE technology must be taken into account for improving reliability and availability. Self-Healing and selfConfiguration schemes can help the network to recover after the detection of a dropped e-node, modifying others e-node transmitting powers or forwarding 
incoming calls to e-nodes with enough resources to establish and maintain user's connections [18].

Regarding service reliability and availability in the core network, several Key Performance Indicators (KPIs) can be defined for assessing the availability and reliability of the LTE end to end solution. In Liem and Mendiratta [19], it is concluded that at least, LTE voice service will meet the current reliability and availability requirements of GSM-R.

\subsubsection{Safety}

Main challenges concerning security and safety issues, are related with the coding and integrity protection of RRC messages and user data. Due to the IP nature of LTE network, the security risks are related with the proper implementation of security protocols for secure IP transportation, such as IPSec to avoid data manipulation in control and user plane. LTE implements mechanisms for mutual authentication of the UE and the network, and also for encoding and checking message integrity in the data communication between terminal and e-NodeB. The IMS architecture provides mechanisms for secure IP data transport and SIP signalisation is also encoded.

\subsection{Deployment considerations}

The deployment consideration concerning the operation and property of LTE network for railway environments is another challenge that must be analyzed.

Three different deployment scenarios can be considered. The first one is the CAPEX one, where the network is property of the infrastructure manager. The railway operator has full control or railway network, although there are high initial capital expenditures to deploy and start operating the network.

Regarding the other two deployment scenarios, an external company deploys the railway network and the railway operator only incurring OPEX costs. In the second scenario, the external company deploying the network is a telecommunication network operator. The main benefit is the economic one, because there are not initial capital expenditures and the network infrastructure will be shared with the network operator, which can deliver its own passenger services. The main challenge for this scenario is the fact that networks resources are shared between railway operator and the telecommunication company. This is a problem in railway environments, where there is a mismatch between core railway services QoS and RAMS requirements, and additional communication services ones.

In the third scenario, the external company deploy network infrastructure only for railway purposes. In this case OPEX costs can be slightly superior to the second scenario ones, but the railway operator has full control of the network.

\subsection{Spectrum considerations}

Frequency operation band selection plays a key role in the network planning and dimensioning process. First issue is related with the cell radius and its economic impact. Higher operating frequencies demand small cell sizes due to adverse 
propagation conditions, thus, more e-NodeBs must be deployed to reach the required coverage levels. In high speed railways, the overlap of adjacent eNodeBs is a key element due to frequent handovers. Overlap areas are function of LTE handover time and the train speed. Therefore, if small cells are deployed, handovers will become very frequent. As a consequence, the signalling overload in the network will increase, reducing the available bandwidth.

\section{Conclusions}

In this paper, the evolution from current GSM-R communication system towards a new railway train radio system has been assessed. LTE is considered to be the natural evolution for GSM-R. Under this scope, we provided the economic and technological factors linked to this evolution process. LTE communication systems must met specific QoS and RAMS requirements. For delivering railway services, while fulfilling QoS requirements, specific functionalities must be supported by LTE standard. We analyzed the top challenges of LTE to become the new railway train radio system. For this purpose, LTE key features to implement railway functionalities, LTE technical challenges in high speed environments, convergence to an all IP network, LTE spectrum harmonization and network deployment considerations, as well as LTE capabilities to meet the service RAMS requirements have been assessed in this paper.

\section{Acknowledgement}

The authors are thankful for the support of the Spanish Ministry of Science and Innovation within the projects TEC2011-28683- C02-01 and IPT-2011-103437000 .

\section{References}

[1] Mandoc, D., "LTE/SAE - The Future Railway Mobile Radio System: Long-Term Vision on Railway Mobile Radio Technologies". Technical Report. UIC, November 2009.

[2] Furuskar, A., Jonsson, T. and Lundevall, M., "The LTE radio interface key characteristics and performance," Personal, Indoor and Mobile Radio Communications, 2008. PIMRC 2008, vol., no., pp. 1-5, 15-18 Sept. 2008.

[3] Barbu, G., "E-Train, Broadband Communications with Moving Trains", Technical Report. UIC, June 2010.

[4] Wootton, S., "Railway Mobile Communication System User Requirements specification", Technical Report. UIC, October 2010.

[5] Ljubic, I. and Simunic, D., "Advanced Speech Call Items for GSMRailway," Wireless Communication, Vehicular Technology, Information Theory and Aerospace \& Electronic Systems Technology, 2009. Wireless VITAE 2009. Vol., no., pp. 131-136, 17-20 May 2009. 
[6] GSM-R Operators Group, "Project EIRENE, System Requirements Specification”, Technical Report v15.1. UIC, June, 2010.

[7] Badard, B., Diascorn, V. and Boulmier, G. et al, "Migration to VoIP over mobile networks: Technical challenges and economic opportunity analysis," Telecommunications Network Strategy and Planning Symposium (NETWORKS), $201014^{\text {th }}$ International, vol., no., pp. 1-7, 27-30 Sept. 2010.

[8] Chen Wu; "User ID provisioning for SIP registration in IMS," Education Technology and Computer (ICETC), vol.2, no., pp. V2-206-V2-210, 22-24 June 2010.

[9] Paisal, V., “Seamless voice over LTE," Internet Multimedia Services Architecture and Application (IMSAA), 2010 IEEE $4^{\text {th }}$ International Conference on , vol., no., pp. 1-5, 15-17 Dec. 2010.

[10] Technical Specification 3G TS 23.216, V11.4, 3GPP Technical Specification Group Services and System Aspects; Single Radio Voice Call Continuity (SRVCC).

[11] Qiwei, W., Guangliang, R. and Jing T., "A soft handover algorithm for TDLTE system in high-speed railway scenario," Signal Processing, Communications and Computing (ICSPCC), vol., no., pp. 1-4, 14-16 Sept. 2011.

[12] L. Luan et al., "Handover Parameter Optimization of LTE System in Variational Velocity Environment", Proceedings of ICCTA2011, International Conference on Computer Theory and Applications, 2011.

[13] Sesia, S., Toufik, I. and Baker, M.,"LTE the UMTS Long Term Evolution from Theory to Practice", $2^{\text {nd }}$ Edition, John Wiley, Great Britain, 2011.

[14] Ren, X., Ren, G. and Le, C., "Carrier frequency offset estimation for PUCCH in high speed train environment", $12^{\text {th }}$ International Conference on Electronic Packaging Technology and High Density Packaging, pp. 1-4, August 2011.

[15] Nonchev, S., Valkama, M. and Hamila, R. , "Effect of high-velocity scenarios on the performance of MIMO LTE packet scheduling", $8^{\text {th }}$ International Multi-Conference on Systems, Signals \& Devices, pp. 1-6, 2011.

[16] A. E. d. N. y. C. (AENOR), “UNE-EN 50126.1:2005: Aplicaciones ferroviarias. Especificación y demostración de la fiabilidad, la disponibilidad, la mantenibilidad y la seguridad (RAMS)" 2010.

[17] Briso-Rodriguez, C., Cruz, J.M. and Alonso, J.I.; "Measurements and Modeling of Distributed Antenna Systems in Railway Tunnels", IEEE Transactions on Vehicular Technology, vol.56, no.5, pp. 2870-2879, Sept. 2007.

[18] 3GPP, "Telecommunication management; Self-Organizing Networks (SON); Self-healing concepts and requirements", 3GPP TR 32.541, version 10.0.0, Release 10, 2011.

[19] Liem, M. and Mendiratta, V., "Mission Critical Communication Networks for Railways", Bell Labs Technical Journal 16(3), 29-46, 2011. 